\title{
SnapQueue: Lock-Free Queue with Constant Time Snapshots
}

\author{
Aleksandar Prokopec \\ École Polytechnique Fédérale de Lausanne, Switzerland \\ aleksandar.prokopec@alumni.epfl.ch
}

\begin{abstract}
We introduce SnapQueues - concurrent, lock-free queues with a linearizable, lock-free global-state transition operation. This transition operation can atomically switch between arbitrary SnapQueue states, and is used by enqueue, dequeue, snapshot and concatenation operations. We show that implementing these operations efficiently depends on the persistent data structure at the core of the SnapQueue.

This immutable support data structure is an interchangeable kernel of the SnapQueue, and drives its performance characteristics. The design allows reasoning about concurrent operation running time in a functional way, absent from concurrency considerations. We present a support data structure that enables $O(1)$ queue operations, $O(1)$ snapshot and $O(\log n)$ atomic concurrent concatenation. We show that the SnapQueue enqueue operation achieves up to $25 \%$ higher performance, while the dequeue operation has performance identical to standard lock-free concurrent queues.
\end{abstract}

Categories and Subject Descriptors E.1 [Data Structures]: Lists, stacks and queues

Keywords queues, lock-free, concatenation, snapshots

\section{Introduction}

Scalability in a concurrent data structure is achieved by allowing concurrent accesses to execute independently on separate parts of the data structure. While efficient concurrent implementations exist for many different data structure types [15], efficient operations that change their global state are still largely unexplored. For example, most concurrent hash tables [7] [13], concurrent skip lists [21], and concurrent queues [14] [22] do not have an atomic snapshot operation, size retrieval or a clear operation.

Permission to make digital or hard copies of all or part of this work for personal or classroom use is granted without fee provided that copies are not made or distributed for profit or commercial advantage and that copies bear this notice and the full citation on the first page. Copyrights for components of this work owned by others than ACM must be honored. Abstracting with credit is permitted. To copy otherwise, or republish, to post on servers or to redistribute to lists, requires prior specific permission and/or a fee. Request permissions from Permissions@acm.org.

SCALA'15, June 13, 2015, Portland, OR, USA

Copyright 2015 ACM 978-1-4503-3626-0/15/06 ...\$15.00

http://dx.doi.org/10.1145/2774975.2774976
Concurrent queues are used as buffers between producers and consumers in streaming platforms, as event queues in reactive programming frameworks, or mailbox implementations in actor systems. Traditionally, concurrent queues expose enqueue and dequeue operations. Augmenting them with atomic global operations opens several new use cases:

- Persisting actor state: Actor frameworks expose persistence modules to persist and recover actor state [1]. Persisting the state requires blocking the mailbox until the pending messages are copied. With an efficient snapshot operation, the mailbox can be persisted in real-time.

- Forking actors: Adding a fork or a choice operator to the actor model [26] requires copying the contents of the mailbox. To achieve atomicity, the corresponding actor is blocked during the copying and cannot access the mailbox. Efficient snapshots help avoid this problem.

- Dynamic stream fusion: Streaming platforms express computations as dataflow graphs. Each node in this graph executes a modular subset of the computation, but also introduces some buffering overhead [24]. Runtime optimizations eliminate this overhead by fusing subsets of the graph. Efficient, atomic buffer concatenation and joining allow optimizations without blocking the computation.

This paper introduces a lock-free queue implementation, called SnapQueue, which, in addition to enqueue and dequeue operations, exposes an efficient, atomic, lock-free transition operation. The transition operation is used to implement snapshots, concatenation, rebalancing and queue expansion. Although SnapQueue is a concurrent data structure, it relies on a persistent data structure to encode its state. We describe the SnapQueue data structure incrementally:

1. We describe lock-free single-shot queues, or segments, and their enqueue and dequeue operations in Section 2.

2. We show that segments can be atomically frozen, i.e. transformed into a persistent data structure.

3. We describe SnapQueues and their fundamental atomic operation called transition in Section 3.

4. We implement SnapQueue enqueue, dequeue, snapshot and concatenation using the transition operation. 


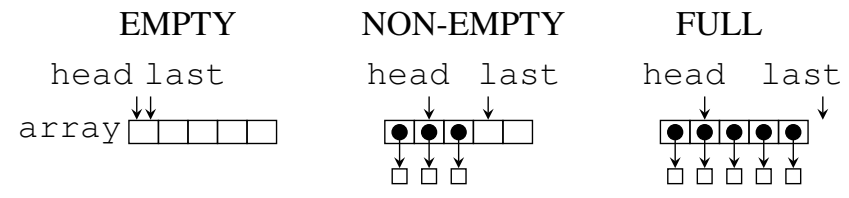

Figure 1. Single-Shot Queue Illustration

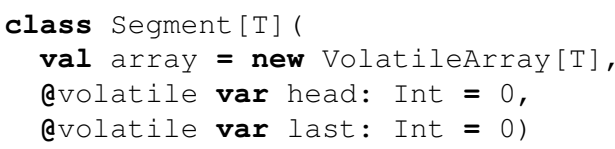

Figure 2. Single-Shot Queue Data Type

5. We show how to tune the running time of SnapQueue operations using persistent data structures in Section 4.

6. We evaluate SnapQueues against similar concurrent queue implementations in Section 5, and show that having snapshot support adds little or no overhead.

The goal of the paper is not only to propose a novel concurrent, lock-free queue with atomic constant time snapshots, but also to reproach the common belief that persistent data structures are slow, and consequently irrelevant for highperformance parallel and concurrent computing. As this paper shows, persistent data structures can simplify the development of and reasoning about concurrent data structures.

For the sake of conciseness, code listings slightly diverge from valid Scala code in several ways. First, atomic READ, WRITE and CAS operations differ from standard Scala syntax, which relies on the Unsafe class. These operations retain the standard Java volatile semantics, and are used to access volatile object fields. Second, volatile arrays are represented with a non-existent volatileArray class. Finally, generically typed methods sometimes accept or return special singleton values. This does not type-check in Scala, but can be worked around at a small syntactic cost. The complete SnapQueue implementation can be found in our online code repository [2].

\section{Single-Shot Lock-Free Queue}

We start by examining a simplistic lock-free queue implementation, called a single-shot lock-free queue. This queue is bounded - it can contain only up to $L$ elements. Second, only up to $L$ enqueue and up to $L$ dequeue operations can be invoked on this queue. Despite these limited capabilities, single-shot lock-free queue is the basic building block of the SnapQueue, as we show in Section 3.

Single-shot queue is defined by a single data type called segment, shown in Figure 2. Segment contains an array of queue elements, initially filled with special EMPTY values, head - the position of the first element in the queue, and last - the estimated position of the first EMPTY array entry.

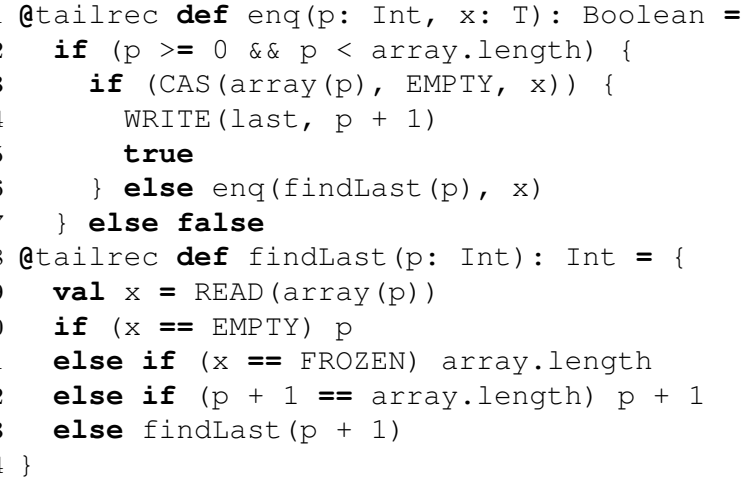

Figure 3. Single-Shot Queue Enqueue Operation

As we will see in Section 2.2, a single-shot queue can become frozen, in which case no subsequent enqueue or dequeue operations can succeed. In this frozen state, array may contain a special FROZEN value.

\subsection{Basic Operations}

In this section, we study the basic single-shot queue operations. The enqueue operation overwrites an EMPTY entry in the array. At all times, it ensures that the array corresponds to the string REMOVED ${ }^{p} \cdot \mathrm{T}^{n} \cdot \mathrm{EMPTY}^{m}$, where $\mathrm{T}$ is the element type, and array length is $L=p+n+m$. After inserting an element, enqueue sets the last field to point to the first EMPTY entry.

We define an auxiliary eng method in Figure 3, which takes an estimated position $p$ of the first EMPTY entry, and an element $x$. The enq first checks that $p$ is within the bounds of array. It then attempts to atomically CAS an EMP TY entry with $x$ in line 3. An unsuccessful CAS implies that another enq call succeeded, so findLast finds the first special entry, and eng is retried. A successful CAS means that $x$ is enqueued, so the value last is increased in line 4. Due to potential concurrent stale writes, last is an underestimate.

The enq precondition is that $p$ is less than or equal than the actual first EMP TY entry position. This is trivially ensured by specifying the last field when calling enq.

Lemma 1. If eng returns true, then its CAS operation added an element to the queue. Otherwise, the queue is either full or frozen.

Dequeue atomically increments the head field to point to the next element in the queue. Unlike last, the head field always precisely describes the position of the first unread element. When head becomes larger than array length, the queue is either empty or frozen. Similarly, when head points to EMPTY or FROZEN, the queue is considered empty or frozen, respectively.

The deq method in Figure 4 starts by reading head to a local variable $p$, and checks if $p$ is within bounds. If it is greater than the array length, the queue is empty. If $p$ is 


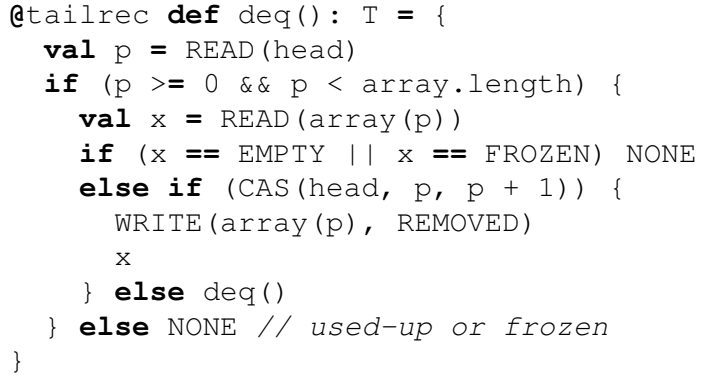

Figure 4. Single-Shot Queue Dequeue Operation

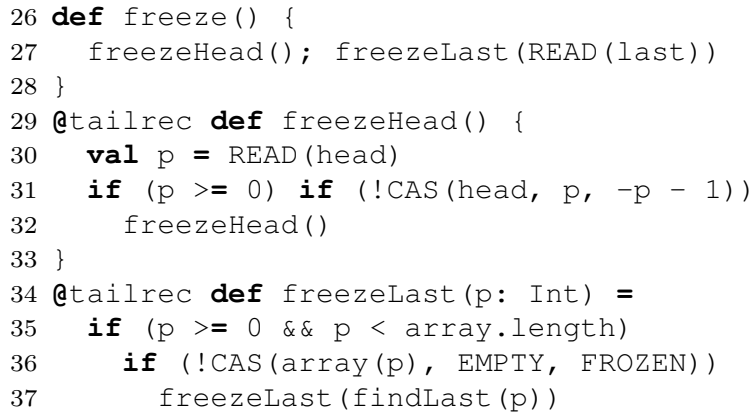

Figure 5. Single-Shot Queue Freeze Operation

negative, the queue is frozen (explained in Section 2.2). If $p$ is within bounds, deq reads the element at $p$ to a local variable $\mathrm{x}$ in line 18. If $\mathrm{x}$ is either EMPTY or FROZEN, then the end of the queue was reached, and deq returns NONE. Otherwise, deq atomically increments head in line 20, or retries the operation when CAS fails. If the CAS in line 20 succeeds, the thread that executed it must eventually remove the element from array in line 21, to avoid memory leaks.

Lemma 2. If deq returns NONE, the queue is either empty or frozen. If deq returns an element, the CAS operation in deq previously incremented head by one.

\subsection{Freeze Operation}

Single-shot queue also exposes the freeze operation, which prevents subsequent updates from succeeding. The method freeze first calls freezeHead, which atomically replaces the head value with a corresponding negative value. This prevents subsequent deq operations. The freeze method then calls freezelast, which enqueues a FROZEN element. This prevents subsequent enq operations.

After the freeze method completes, the segment object becomes immutable - no operation will subsequently change its state. A stale write may change the last field, but the logical state is only defined by the array and head.

Lemma 3. After freeze returns, subsequent operations can only modify the last field and the array entries preceding the position -head - 1 .

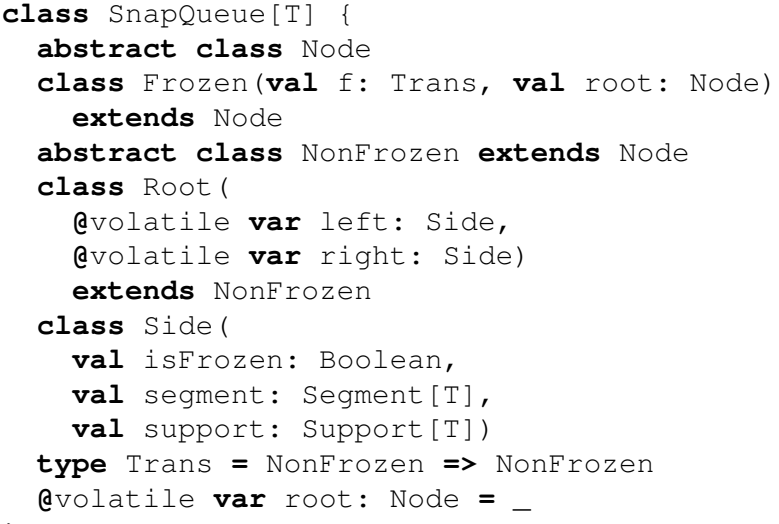

Figure 6. SnapQueue Data Types

Lemma 4. When freeze returns, array either contains no EMPTY entries, or contains a single FROZEN entry. In both cases, head is negative.

Note that, due to the findLast call, complexity of the freeze operation is $O(L)$, where $L$ is the length of the array.

\section{SnapQueue}

Single-shot queue, or segment, is simple, and consequently efficient. However, boundedness and the $L$-operations limit make it uninteresting for most practical purposes. Our goal is an unbounded queue with arbitrary atomic operations, which we name SnapQueue. In this section, we show its data types.

When SnapQueue size is less than $L$, the elements are stored as a segment. SnapQueue overcomes segment's $L$ operations limit by reallocating the segment when it becomes full. To overcome boundedness, SnapQueue uses a secondary representation: a segment-support pair. When the number of elements exceeds $L$, the segment is replaced by two segments left and right. Subsequent enqueue operations use the right segment, and dequeue operations use the left segment.

If there are more than $2 L$ elements, additional segments are stored in support data structures, hence the name segment-support pair. The support data structure is persistent and kept in the support field. Support data structure must be such that pushing and removing segments retains the order in which the segments were pushed, i.e. a sequence.

The snapqueue [T] class is shown in Figure 6. It has a volatile field root of the Node type. Node is a supertype of segment from Section 2, and of the Root and Frozen types. Segment and Root have another common supertype called NonFrozen. The Root comprises two volatile fields left and right, pointing to immutable segment-support pairs of type side. Their isfrozen field denotes whether Side is frozen (explained in Section 3.1). The support field stores a persistent data structure of the Support [ $T$ ] type, which stores intermediate segments. SnapQueue correctness 

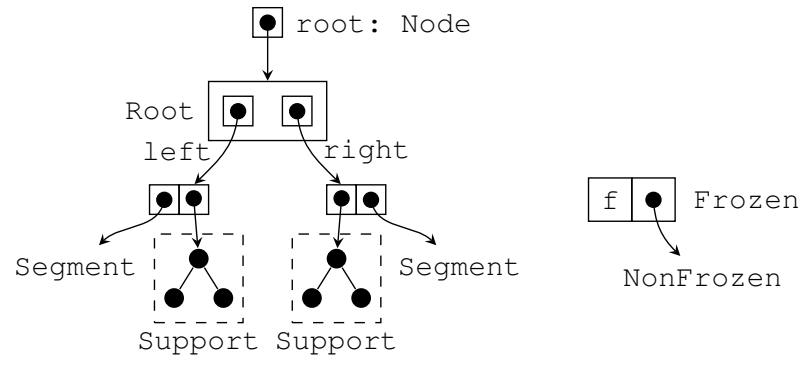

Figure 7. SnapQueue Illustration

does not depend on the choice of this data structure, so we defer discussion on Support [ $\mathrm{T}]$ until Section 4. A particular SnapQueue instance containing a Root is shown in Figure 7.

The Frozen data type contains a reference to either a Root or a segment, and denotes that the underlying SnapQueue is currently in the process of being frozen, or is already frozen. It also contains a transition function of type NonFrozen $\Rightarrow>$ NonFrozen.

As hinted earlier, SnapQueue uses an atomic transition operation, which freezes the SnapQueue before replacing its contents. In the next section, we study how freezing works.

\subsection{Freeze Operation}

Instead of starting with the basic queue operations as in Section 2, we first describe the freeze operation, which is a prerequisite for the transition operation, shown later.

As with the single-shot queue, invoking freeze turns a concurrent data structure into an immutable data structure. The goal of freeze is to invalidate subsequent writes on volatile fields in the SnapQueue. To do this, freeze first freezes the root, then freezes the two side references, and at the end freezes the underlying segments.

The snapqueue\#freeze method in Figure 8 takes the reference $r$ to the previous SnapQueue root. It also takes the transition function, which is explained in Section 3.2 - for now we treat it as extra payload in the Frozen object.

The freeze operation first attempts to replace the expected root $r$ with a fresh Frozen object. If some other thread already changed root to a value different than $r$, freeze signals failure by returning null. Otherwise, if the CAS changes root to the Frozen object, completeFreeze is called to freeze the left and right side, and the segments.

Note that, unlike freeze on the single-shot queue from Section 2.2, freeze operation in Figure 8 may fail and return null. In this case, it is up to the caller to retry.

However, if the CAS in line 42 succeeds, the SnapQueue is eventually frozen, since other operations will never modify frozen left and right fields, or frozen segments.

Lemma 5. After freeze returns a non-null value, subsequent operations may only modify the last fields and the array entries preceding -head - 1 in the two segments.

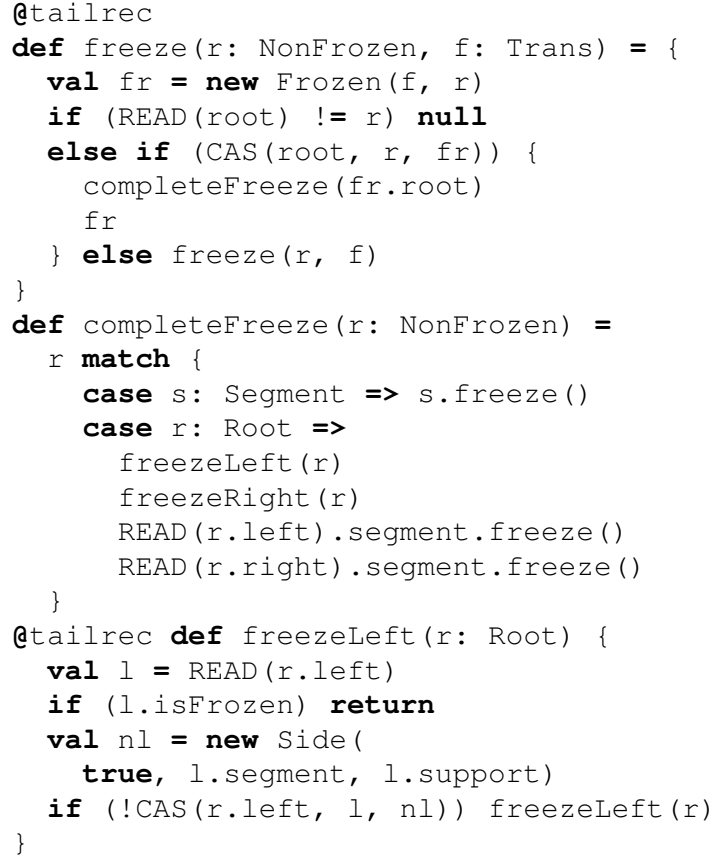

Figure 8. SnapQueue Freeze Operation

Furthermore, if a segment is frozen, then so are the left and right fields.

Proof. This follows from Lemma 3, and the fact that basic SnapQueue operations fail if side is frozen.

Lemma 6. If freeze returns a non-nul I value $f x$, then the value of root atomically changed from $r$ to $f r$, where $r$ is the specified root reference.

The complexity of the freeze operation in this Section is again $O(L)$, where $L$ is the length of the array in segment objects. Importantly, complexity does not depend on the size of the persistent support data structure.

\subsection{Transition Operation}

The transition operation is the most important SnapQueue operation. It atomically exchanges the contents and the structure of the SnapQueue. It is analogous to the CAS operation, with the difference that transition operates on the entire data structure, rather than a single memory location.

The transition operation first freezes the SnapQueue. If freeze fails, then so does transition. However, if freezing succeeds, transition uses a transition function that maps a frozen SnapQueue into a new SnapQueue.

The snapqueue\#transition method in Figure 9 takes the expected root value $r$, and a transition function $f$. After freezing, it invokes completeTransition, which computes the new root with $f$, and atomically replaces the old root with the output of $f$ in line 79 . The helpTransition method is 


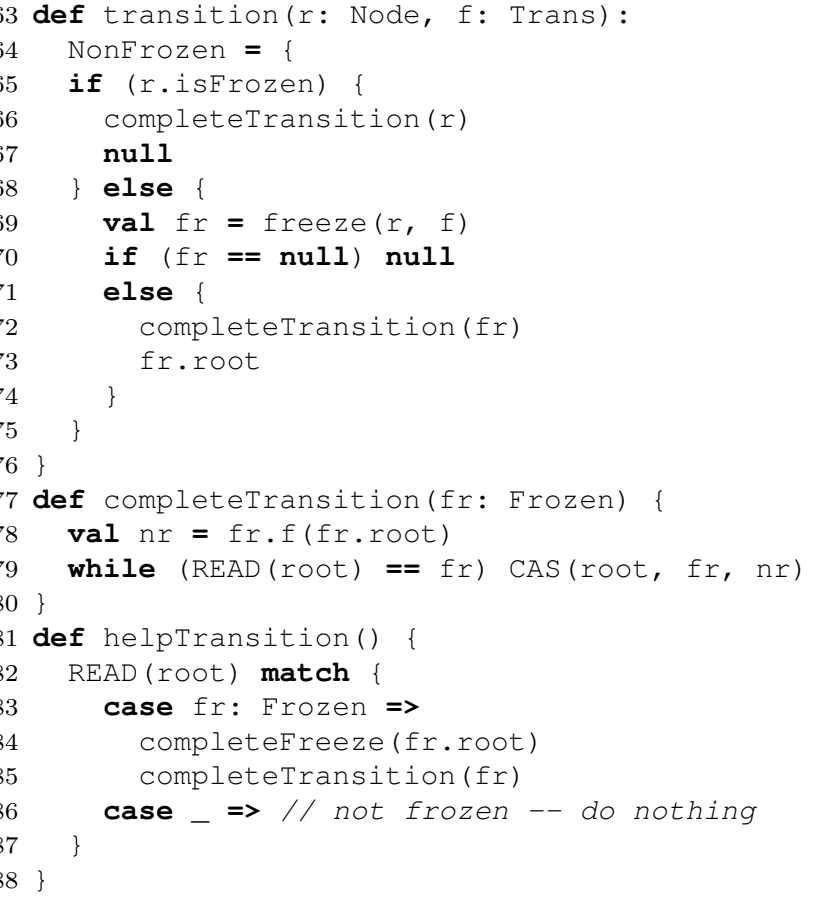

Figure 9. SnapQueue Transition Operations

similar, but gets called by threads executing their separate operations, to help complete the transition in a lock-free way.

Lemma 7. If transition returns a non-null value, then the value of root atomically changed from Node reference $r$ to Frozen $(r, f)$, and then atomically to $f(r)$, where $r$ is the specified root and $f$ is the transition function.

Proof. This is a consequence of Lemmas 5 and 6.

Lemma 8. The transition running time is $O(L+f(n, L))$, where $O(f(n, L))$ is the transition function running time with respect to the support data structure size n, and segment length $L$.

Proof. This is a consequence of the $O(L)$ complexity of the freeze operation, and the fact that the transition function is invoked at least once.

\subsection{Transition Functions}

The transition operation takes a referentially transparent transition function. The transition function is invoked by the time the Node is already frozen, and effectively a persistent data structure, Therefore, the transition can be reasoned about functionally, separate from concurrency concerns.

We consider some concrete transition functions in Figure 10. Assume that the SnapQueue represented with a segment of length $L$ needs to be replaced with a Root, as outlined at the beginning of Section 3. If the SnapQueue contains

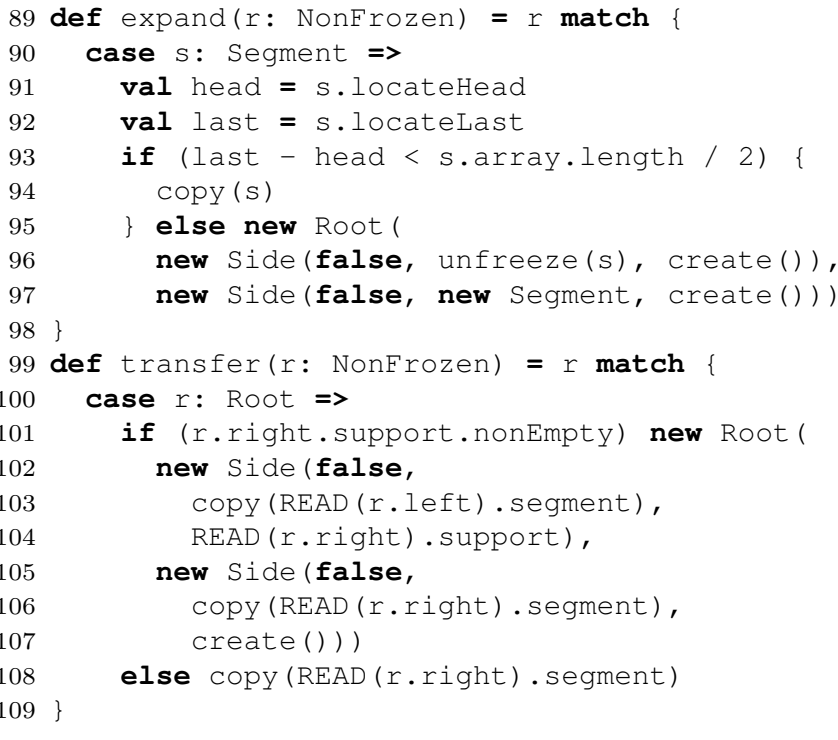

Figure 10. SnapQueue Transition Functions

less than $L / 2$ elements, the expand transition function creates another segment - copy in line 94 copies a frozen segment into a freshly allocated one. If the size is above $L / 2$, expand creates a Root object - here, create allocates an empty support data structure, and unfreeze reallocates the segment. The $L / 2$ approach prevents eagerly alternating between Root and segment representations.

The transfer transition function is used when a Root queue runs out of elements in its left segment and support structure. It transfers the support structure from the right to the left side of the SnapQueue. In Section 3.4, we will see usages of both transfer and expand.

Lemma 9. The running time of both expand and transfer is $O(L)$, where $L$ is the segment size.

Proof. This is the consequence of calling unfreeze and copy, which take $O(L)$ time for an $L$-element segment.

\subsection{Basic Operations}

In this section, we closely examine enqueue and dequeue. For efficiency, SnapQueue basic operations in most cases only modify a segment. Occasionally, a segment is used up and replaced with a new segment from the support structure.

We define the top-level snapqueue\#enqueue method, and three internal, dynamically dispatched enqueue methods on the Root, Frozen and Segment data types. After the top-level enqueue in Figure 11 reads the root, it calls an internal enqueue. Since SnapQueue is an unbounded data structure, it must always be possible to enqueue a new element. If an internal enqueue returns false to indicate that the element was not added, the operation is retried.

The enqueue on the Root type reads the right segmentsupport pair, and the segment's last field. It then calls eng 


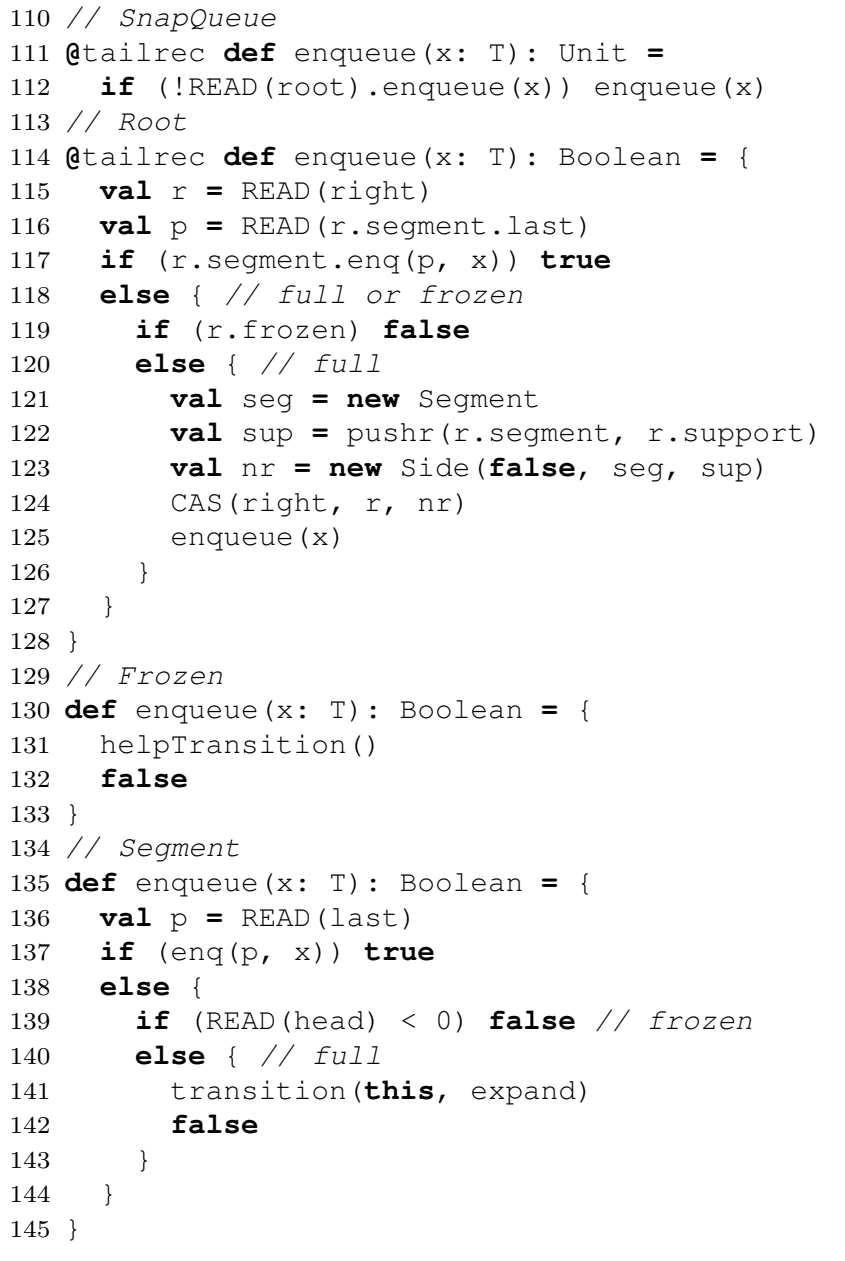

Figure 11. SnapQueue Enqueue Operation

in line 117. If enq returns false, the segment is either full or frozen, by Lemma 1. In line 119, we rely on Lemmas 4 and 5 to check if the segment is frozen. By Lemma 5 , if the segment is frozen, the Root is also frozen, so we return to the top-level enqueue in line 119, and then help complete the transition. Otherwise, if the segment is full, we push its array into the support structure with a persistent pushr operation, and replace the right side with the new side object.

The enqueue on the Frozen type helps the current transition operation to complete, and then retries the enqueue. The enqueue on the segment type calls enq - if the segment is full, it attempts to replace the segment by calling the transition operation from Section 3.2 with the expand function from Section 3.3 before retrying.

The dequeue operation in Figure 12 is similarly separated between the top-level snapqueue\#dequeue, and internal dequeue methods on Root, segment and Frozen types. Root\#dequeue starts by calling deq on the left segment. If deg fails, the segment is either empty or frozen, by Lemma 2 . If the segment is frozen, there is possibly an ongoing tran-

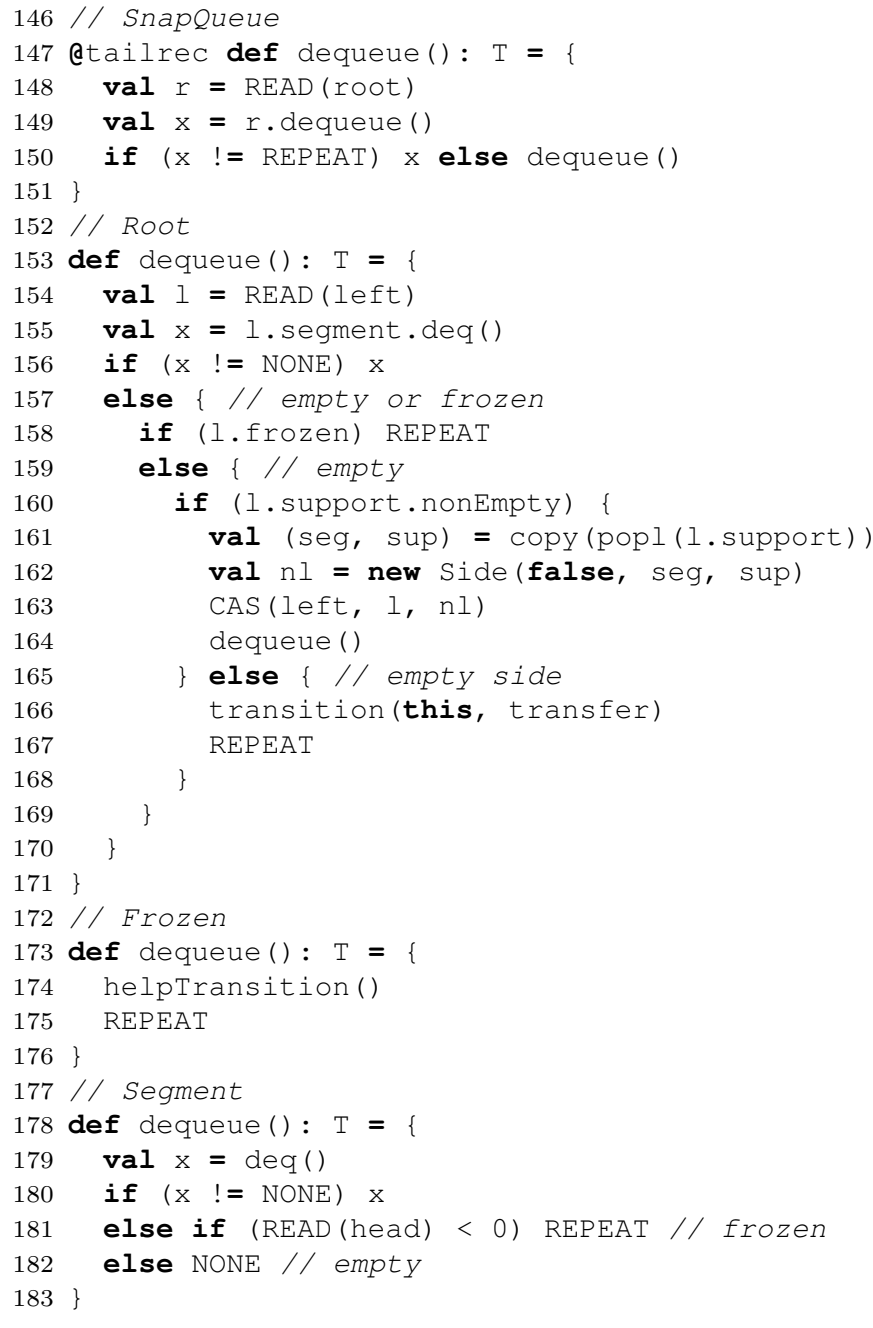

Figure 12. SnapQueue Dequeue Operation

sition, so control returns to the top-level dequeue. Otherwise, if the support data structure is non-empty, dequeue invokes popl to extract an array for a new segment, refreshes the left side, and retries. If the support is empty, segments must be borrowed from the right side, so transition with the transfer function from Section 3.3 is called.

We can now state the following two theorems. The first theorem establishes the operation running time.

Theorem 1 (SnapQueue Running Time). Let $L$ be the length of the segment array. The amortized running time of the enqueue operation is $O\left(L+\frac{f(n)}{L}\right)$, where $O(f(n))$ is the running time of the pushr operation of the support structure containing $n$ elements. The amortized running time of the dequeue operation is $O\left(L+\frac{g(n)}{L}\right)$, where $O(g(n))$ is the running time of the pop I operation of the support structure containing $n$ elements.

The second theorem establishes the contention rate between producers and consumers as the number of operations 


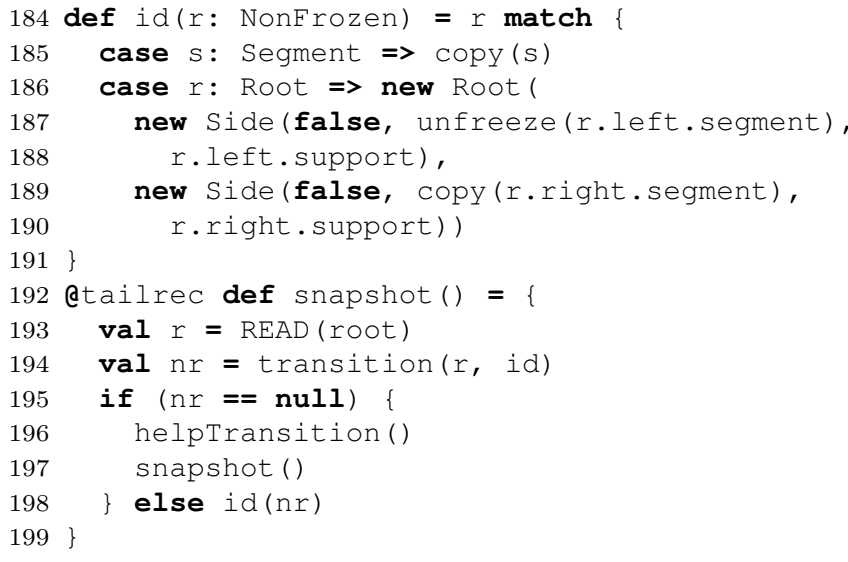

Figure 13. SnapQueue Snapshot

between writes to the root field - the higher this value, the lower the contention. When the consumer periodically exhausts the support data structure in the left side object, the root is frozen to call transfer. When this happens, the producers and consumers contend. We care about this, since typical actor systems have a single consumer and many producers - for example, multiple producers should not be able to flood the single consumer as a consequence of contention.

Theorem 2 (SnapQueue Contention). Let $L$ be the length of the segment array, $O(g(n))$ the running time of the pop I operation, and $n$ the total number of elements in the SnapQueue. In a sequence of basic operations, there are on average $O\left(L+\frac{n \cdot g(n)}{L}\right)$ dequeue operations between two root field writes (that is, between two freeze operations).

Proof. Both theorems are a consequence of Lemmas 8 and 9, and implementations in Figures 11 and 12.

\subsection{Snapshot and Concatenation}

The transition operation from Section 3.2 is very expressive, as it atomically changes the state of the SnapQueue given an arbitrary transformation. In this section, we use it to implement atomic snapshots and atomic concatenation.

The snapshot method in Figure 13 uses the identity transition function $i d$. This method repetitively invokes the transition operation until it becomes successful. It is easy to see that the snapshot running time is $O(L)$.

Next, we note that the Lemma 7 implies the following:

Lemma 10. If the value returned by the transition function $f$ gets written to the root field by transition, then during the corresponding invocation of $f$, SnapQueue was frozen.

We rely on Lemma 10 to implement atomic concatenation. To achieve atomicity, this operation must simultaneously freeze two SnapQueues. Note that two concatenation operations on the same pair of SnapQueues could potentially deadlock if they freeze the SnapQueues in the oppo-

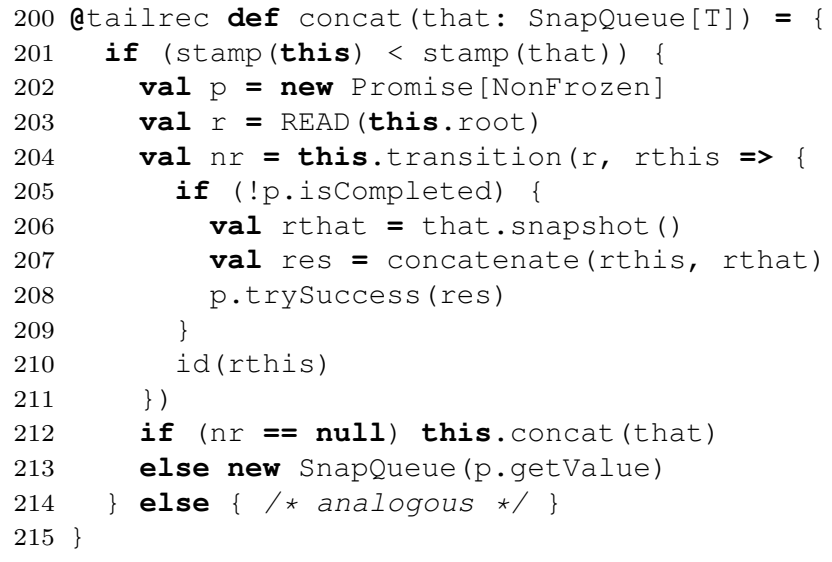

Figure 14. SnapQueue Concatenation

site orders. To prevent this, we need to establish an ordering between SnapQueue instances - we assume that a stamp method associates unique integers to each queue.

The snapqueue\#concat operation in Figure 14 first calls stamp to establish the order. We assume this comes before the argument that - the reverse case is analogous. The concat method creates a new Promise object [9], which serves as a placeholder for the resulting SnapQueue. The Promise object is a single-assignment variable - it can be assigned a value at most once using the trysuccess method. After concat starts a transition on this SnapQueue, it creates a snapshot of that SnapQueue. At this point, we have two frozen data structures and can concatenate them with a persistent concatenate operation in line 207. The result is stored into the Promise in line 208.

Assume that the transition returns a non-null value. This can only happen if some thread assigned the result into the Promise before transition returned. That thread called snapshot on that before writing to the Promise. By Lemma 10, this SnapQueue was frozen at the time, implying that the concatenation operation is atomic with respect to both SnapQueues.

\section{Support Data Structures}

In Section 3, we introduced SnapQueue, which relies on a persistent sequence data structure, called support, to store arrays of size $L$. Since the running time of SnapQueue operations depends on the running time of the support data structure, SnapQueue constitutes a framework for assessing different persistent data structure implementations. In this section, we consider support structures that provide the following operations: create, which creates an empty support structure, nonEmpty, which checks if the support structure is empty, pushr, which appends the element on the right side, popl, which removes the leftmost element, and concatenate, which concatenates two support structures. 


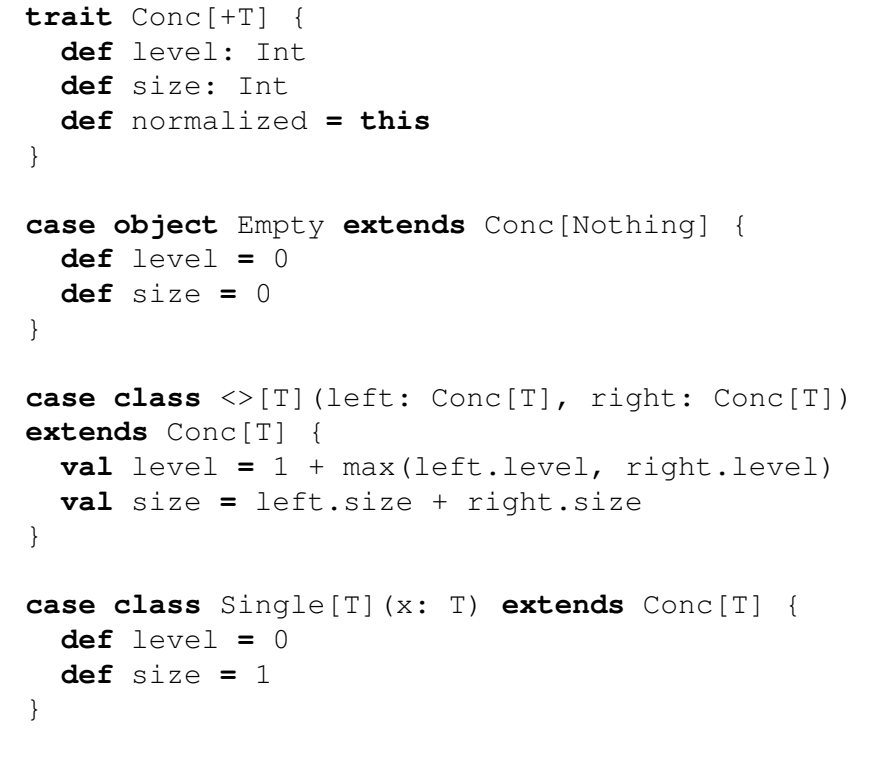

Figure 15. Conc-Tree Data Types

When choosing a data structure, we usually consider the following properties. First, asymptotic running time with respect to the data structure size should be as low as possible, in the ideal case $O(1)$. Second, constant factors must be low to ensure good absolute running time. With high constant factors, even an $O(1)$ operation can be prohibitively slow for typical data structure sizes. Finally, the data structure should be simple - it should be easy to comprehend and implement.

Although persistent data structures with worst-case $O(1)$ implementations of the desired operations exist [12], their implementations are not simple, and have high constant factors. We know from Theorem 1 that the SnapQueue operations run in $O\left(L+\frac{f(n)}{L}\right)$ time. To optimize SnapQueues, we must optimize $L$ against the support structure whose pushr and popl operations are $O(1)$ with low constant factors.

In the rest of this section, we study Conc-Trees [17] as the support data structure. Conc-Tree is a particular implementation of the conc-list abstraction from Fortress [5] [25], originally intended for functional task-parallel and data-parallel programming. Conc-Tree concatenation is $O(\log n)$, but its $O(1)$ popl and pushr are simple and efficient.

The Conc abstract data type is shown in Figure 15. This data type specifies the size of the tree, i.e. the number of elements, and the level, i.e. the tree height. A Conc is either an Empty tree, a tree with a single element, or an inner node $<>$ (pronounced conc) with two subtrees. Conc defines a method normalized, which returns the tree composed only of these three data types.

Conc-Trees maintain the following invariants. First, the Empty tree is never a child of other nodes. Second, the absolute level difference of the children in $<>$ nodes is smaller than or equal to 1 . This ensures balanced Conc-Trees - longest and shortest paths differ by at most $2 \times$.

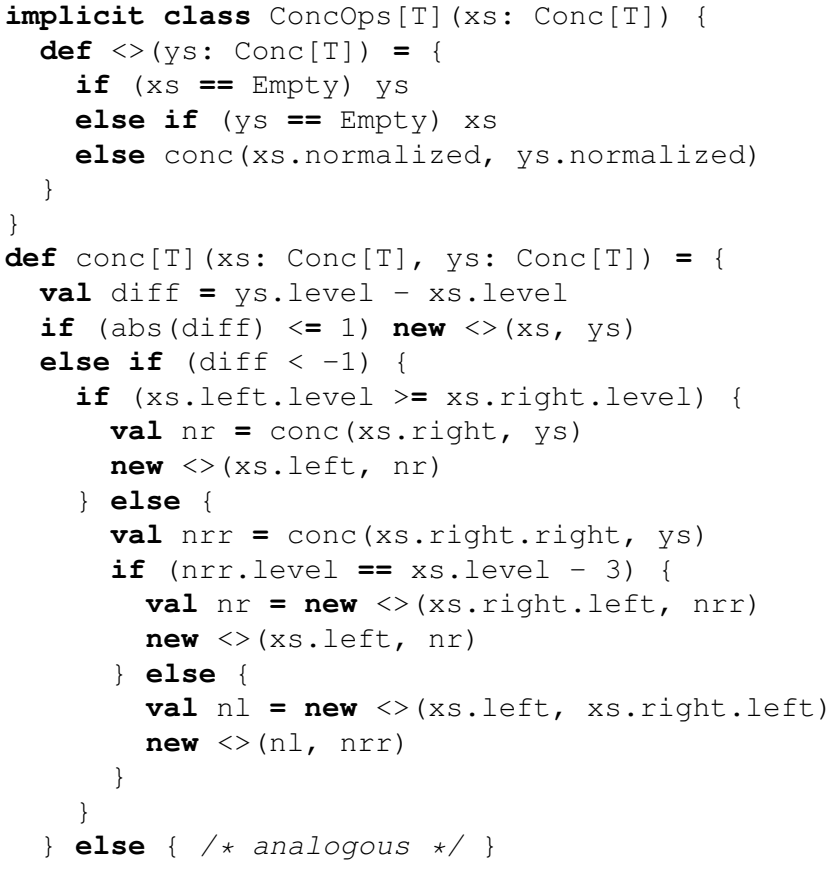

Figure 16. Conc-Tree Concatenation

In Figure 16, we implement concatenation for ConcTrees. Similar to how the symbol of the Scala : : data type (pronounced cons) is used to prepend to a List, we borrow the $<>$ data type symbol for Conc concatenation. Thus, the expression new $<>$ (xs, ys) links the two trees $x s$ and ys by creating a new $<>$ object, whereas $x \mathrm{~s}<>$ ys creates a balanced Conc-Tree that is a concatenation of $x s$ and $y s$.

The public $<>$ method eliminates Empty trees before calling the recursive conc method. The conc method links trees if the invariants allow it. If they do not, conc concatenates the smaller Conc-Tree with a subtree in the bigger ConcTree, before re-linking the result. The conc running time is $O\left(\left|h_{x s}-h_{y s}\right|\right)$, where $h_{x s}$ and $h_{y s}$ are the tree heights [17].

The $<>$ method is sufficient for pushr:

def pushr(xs: Conc $[T], x: T)=x s<$ Single $(x)$

Unfortunately, this implementation is $O(\log n)$, and our goal is to achieve constant time. We will next extend the basic Conc-Tree data structure to achieve this goal.

In Figure 17, we introduce a new type Ap, which is isomorphic to the $<>$ data type. The distinction with the Ap type is that its subtrees do not need to differ in level by at most 1 . Instead, Ap introduces two new invariants. First, an Ap node can only be the left subtree of another Ap node. Otherwise, an Ap must be the root of the tree. Second, if an Ap node $n$ has a Ap node $m$ in its left subtree, then $n$. right. level must be strictly smaller than m.right. level.

As a consequence of the two Ap invariants, Conc-Trees that contain Ap nodes correspond to to numbers in the binary 


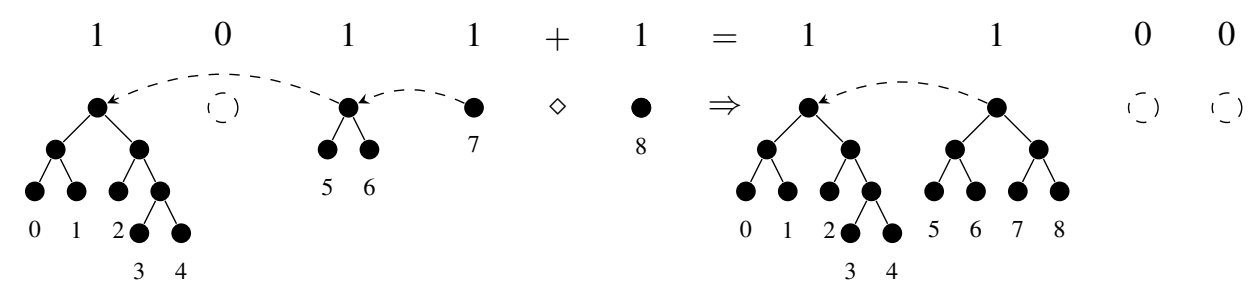

Figure 18. Correspondence Between the Binary Number System and Conc-Trees

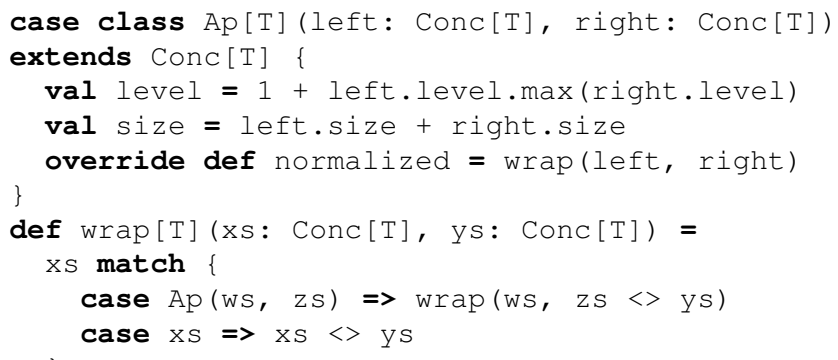

Figure 17. Conc-Tree Append Data Type

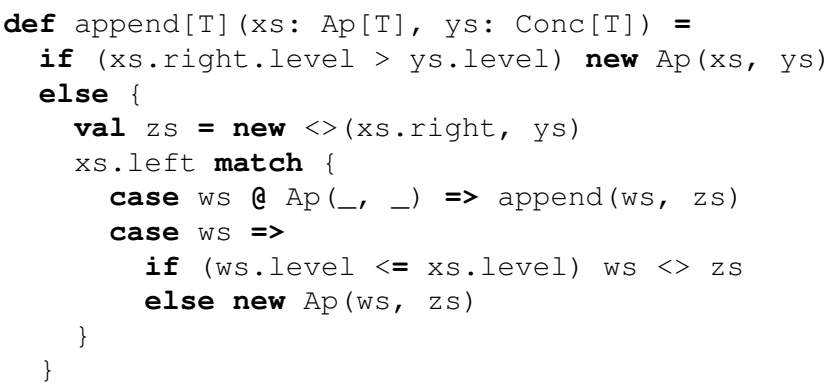

Figure 19. Conc-Tree Append Operation

system, as shown in Figure 18. Each Ap node $\mathrm{n}$ corresponds to a digit whose position is equal to the height $n$.right. Absence of an Ap node with a specific height indicates the absence of the corresponding digit.

This correspondence has two consequences. First, since we know how to increment a binary number by adding a single digit on the right, we can equivalently append single trees by linking trees of the same height together [16]. Second, since incrementing a number in the binary number system on average takes $O(1)$ computational steps, appending a single tree also takes $O(1)$ steps on average.

This is illustrated in Figure 18, where a Single tree is appended to a Conc-Tree corresponding to the binary number 1011. Appending triggers a chain of carries, which stops upon reaching 0 -digit. The append method that implements this is shown in Figure 19, and it is used to directly implement the pushr operation:

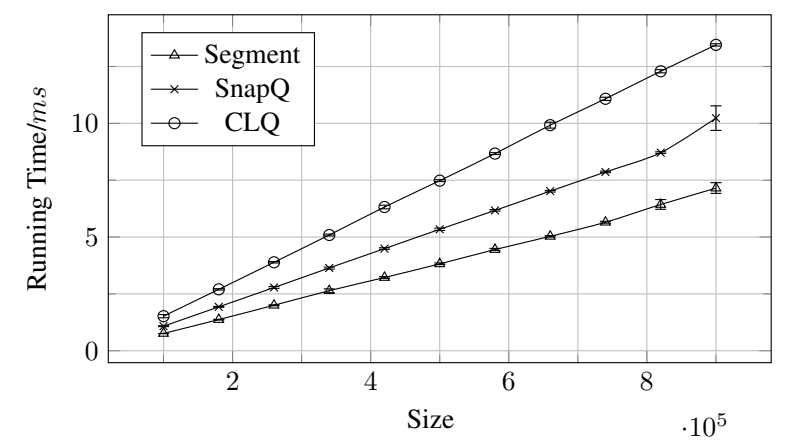

Figure 20. 1-Thread Enqueue, $\mathrm{L}=128$

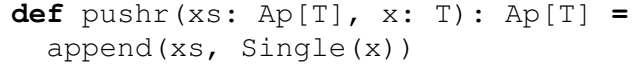

The append implementation is similar to functional random access lists [16]. Both achieve amortized $O(1)$ appends using a number system representation. However, Conc-Trees with Ap nodes can additionally be concatenated in $O(\log n)$ time. For this, an Ap tree must first be normalized - transformed into a tree composed only of single and $<>$ nodes. This is the task of the wrap method in Figure 17, which concatenates all the right subtrees in the append list. Since concatenation takes $O\left(\left|h_{x s}-h_{y s}\right|\right)$ time, and the sum of consecutive height differences between the right subtrees is $O(\log n)$, the wrap method runs in $O(\log n)$ time [17].

To conclude, we defined a persistent data structure with amortized $O(1)$ pushr, and $O(\log n)$ concatenation. Although we did not show it in this section, we can similarly define a Prep node to obtain a $O(1)$ popl.

\section{Evaluation}

In this section, we experimentally evaluate optimal segment lengths, and compare the SnapQueue against the JDK Concurrent LinkedQueue (CLQ) implementation based on Michael and Scott's concurrent queue [14]. The benchmarks are performed on an Intel 2.8Ghz i7-4900MQ quad-core processor with hyperthreading. We use the ScalaMeter tool to execute our benchmarks [18], which relies on standard JVM benchmarking methodologies [8].

The workload in the following benchmarks comprises of either enqueuing or dequeuing size elements, spread across some number of threads. When we study single-thread performance, we vary the size on the $x$-axis. Otherwise, we 


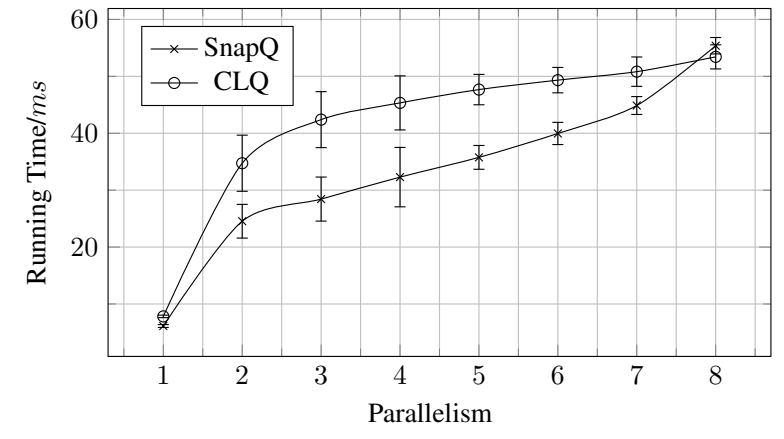

Figure 21. N-Thread Enqueue, size $=500 k, \mathrm{~L}=128$

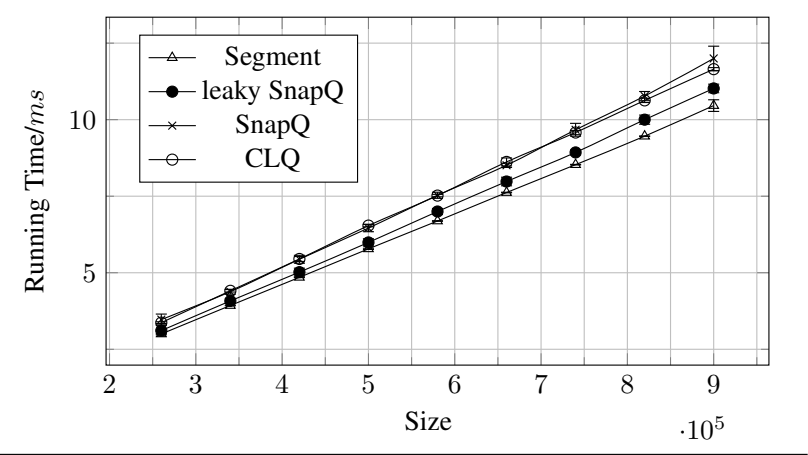

Figure 22. 1-Thread Dequeue, $\mathrm{L}=128$

vary the number of threads and keep the total number of elements size fixed. The $y$-axis shows the running time. The benchmarks test the two data structures in extreme conditions. In practical applications, producers and consumers perform some work between enqueue and dequeue calls, but here they exclusively call queue operations.

In Figure 20, we compare the single-thread running time of SnapQueue enqueue method against the running time of the add method of CLQ. The enq method of the segment class is almost $2 \times$ smaller than that of CLQ. This speedup is due to the extra cost of allocating a Node object that CLQ pays when enqueuing an element. With $L=128$, SnapQueue enqueue performance is around $25 \%$ better than CLQ - the performance loss with respect to segment is due to additional atomic reads of root and right fields, intermediate method calls, and Conc-Tree manipulations.

In Figure 21, we show the same workload distributed across $P$ threads, where $P$ varies from 1 to 8 . This benchmark shows that CLQ contention is larger than SnapQueue contention for fewer threads, but the two data structures have similar contention at higher parallelism levels.

Next, we show single-thread dequeue performance in Figure 22. Here, SnapQueue and CLQ have almost identical running times - CLQ's poll method is faster than the add method from Figure 20, since poll does not have to allocate any objects. Calling deq on segment is about $15 \%$ faster.

The leaky SnapQ is a version of SnapQueue with a slightly faster dequeue operation. In line 21 of the listing

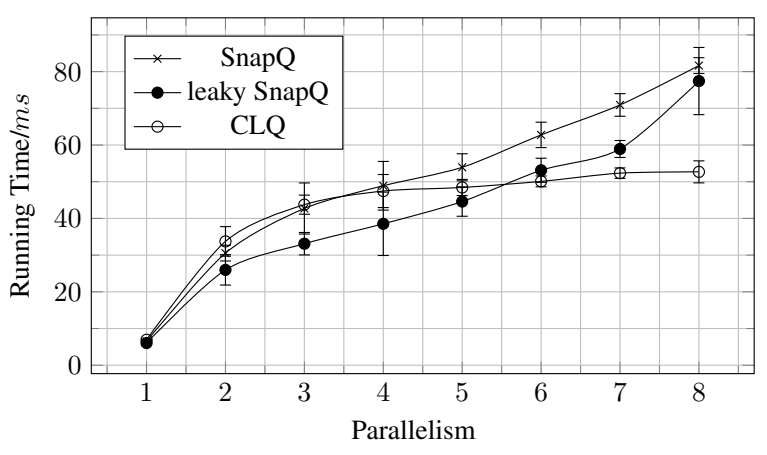

Figure 23. $\mathrm{N}$-Thread Dequeue, size $=500 k, \mathrm{~L}=128$

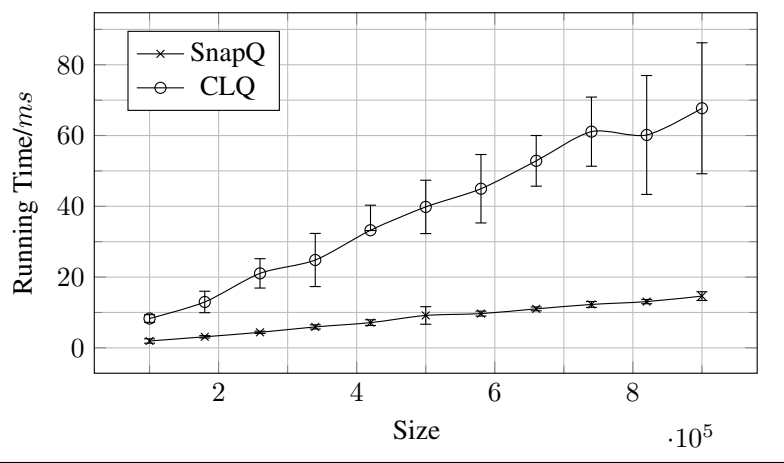

Figure 24. 1 Producer, 1 Consumer

in Figure 4, we used an atomic WRITE operation to replace the logically removed element with a special REMOVED value. This ensures that the SnapQueue does not keep references to dequeued elements in the leftmost segment, and avoids a potential memory leak. The WRITE in the dequeue operation mutates arrays that come from the support data structure. Since arrays in the support data structure, due to operations such as snapshot, can be shared between multiple copies of the SnapQueue, dequeue must copy the array returned by popl in line 161 of the listing in Figure 12.

Some applications are unaffected by memory leaks, either because the objects are small, or because the dequeue operation is called often. In such applications, the WRITE in line 21 is unnecessary. Consequently, the copy call in line 161 can be removed. This improves performance of the dequeue operation by about 10\%, as shown in Figures 22 and 23.

In Figure 23, we show the same workload spread across multiple threads. The contention effects are similar between SnapQueue and CLQ when $P \leq 4$. For $P>4$, SnapQueue contention-related slowdowns are larger than those of CLQ. We believe that this is due to increased amount of allocations that occur when multiple threads simultaneously attempt to replace the leftmost segment after the popl in line 161 .

Next, we test for contention between a single producer and a single consumer in Figure 24. Here, the producer starts by enqueuing size elements, and the consumer simultaneously dequeues them. While in this benchmark SnapQueue shows almost the same performance as in Figure 22, CLQ 


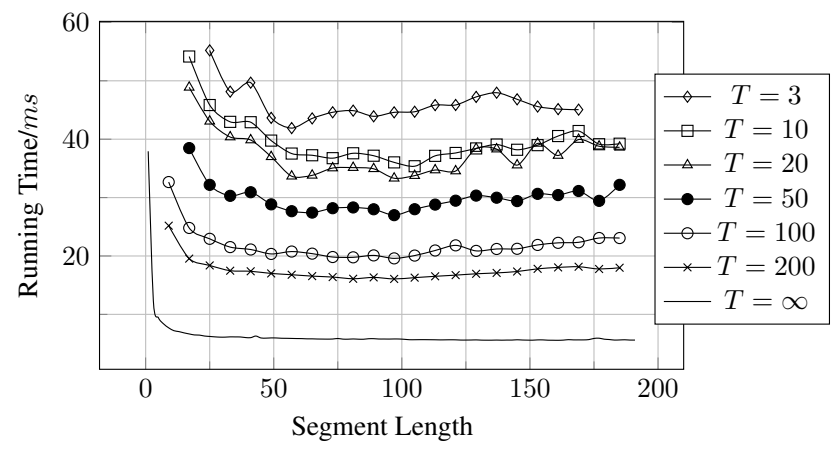

Figure 25. Running Time vs Segment Length

suffers from contention. We postulate that this is because the CLQ poll method is faster than the add method, so the consumer repeatedly catches up with the producer, causing contention. This effect is important only for those applications in which producers and consumers spend most of the time invoking queue operations.

For the purposes of the last benchmark, we recall the Theorem 1, where we established that the running time of queue operations is bound by $O\left(L+\frac{f(n)}{L}\right)$, where $L$ is the segment length, and $O(f(n))$ is the complexity the support data structure operations. Every queue operation can be interrupted with a snapshot, in which case two segments of length $L$ must be copied, hence the first term. Then, after every $L$ operations, a segment must be pushed or popped from the support structure, hence the second term $\frac{f(n)}{L}$. Assuming that snapshots occur periodically at some rate $T$, the running time becomes $O\left(\frac{L}{T}+\frac{f(n)}{L}\right)$. Optimizing the SnapQueue amounts to optimizing this expression for some period $T$.

Figure 25 shows the dependency between the running time of the enqueue method and the SnapQueue segment length for different choices of the period $T$. For $T=\infty$, that is, when snapshots do not occur, the first term becomes 0 and the corresponding curve converges around $L=50$, but has no optimum. For other values of $T$, the optimum value appears somewhere between $L=64$ and $L=128$. For $T=3$, that is, when snapshots occur very frequently, the first term becomes dominant and the optimum shifts to around $L=64$. Based on these results, the recommended SnapQueue segment length is 64 for most applications.

\section{Related Work}

Lock-free concurrent data structures are an active area of research, and there exist extensive concurrent data structure overviews [10] [15]. Here we focus on the related concurrent queues and data structures with snapshots.

Original lock-free concurrent queues allocate a separate node per each enqueued object [14]. SnapQueue avoids this by allocating a chunk of memory (segment), and stores the objects within. As a result, SnapQueue has decreased memory footprint compared to traditional lock-free queues.
The single-shot lock-free queue is similar to the FlowPool data structure [20], which also allocates memory chunks instead of single nodes per element. FlowPool basic operations use two CAS operations instead of one, and are more complex. FlowPools can also reduce contention at the cost of weakened ordering guarantees [23], but do not have atomic snapshots. Disruptor [27] is bounded concurrent ring-buffer data structure designed to serve as a highperformance buffer. Its memory is also allocated in chunks for better cache locality, and decreased memory footprint.

Some concurrent data structures have in the past provided efficient snapshots. SnapTree [6] is a concurrent AVL tree that provides a fast clone operation and consistent iteration. Ctrie [19] [20] is a concurrent hash trie implementation with constant time atomic lazy snapshots, atomic clear operation and consistent iteration. Similar to how SnapQueue uses a persistent data structure to efficiently switch between global states, Ctrie relies on a persistent data structure to implement its lazy snapshot operation. A different approach in the past was to provide a general framework for snapshots [4] although this is a general lock-free snapshot technique, these snapshots are generally $O(n)$ in the size of the data structure.

An extensive overview of traditional persistent data structure design techniques is given by Okasaki [16]. Binary number representation used by Conc-Trees is inspired by random access lists, which represent data as a list of complete binary trees. In this regard, Conc-Trees are unlike most traditional immutable sequence data structures, where every binary digit of weight $W$ corresponds to a complete tree with $W$ elements, but instead rely on relaxed balancing typically used in AVL trees [3]. This combination of features allow Conc-Trees to retain logarithmic concatentation along with amortized constant time prepend and append operations.

Conc-list abstraction appeared in Fortress [5], where it was used for task-parallel programs [25]. More advanced Conc-Tree variants achieve worst-case $O(1)$ prepend and append [17], but are more complicated. Some persistent trees achieve amortized $O(1)$ [11] and worst-case [12] $O(1)$ concatenation, at the cost of high implementation complexity.

\section{Conclusion}

We presented the SnapQueue data structure - a lock-free, concurrent queue implementation with atomic global transition operation. This transition operation is the building block for enqueue and dequeue operations, as well as atomic snapshots and concatenation. We note that transition operation is not limited to these applications, but can be used for e.g. atomic clear, size retrieval or a reverse operation. SnapQueue stores its state in a persistent support data structure, and the transition operation leverages this to improve performance.

Although we did not explicitly show lock-freedom, we note that lock-freedom can be easily proved by showing that each failing CAS implies the success of another concurrent 
operation, and that each state change is a finite number of instructions apart.

We analyzed the running time and contention. This analysis shed light on several important design ideas. First, the contention between the producers and the consumers is an inverse function of the number of elements in the queue. Second, the cost of periodic segment reallocation is amortized by the segment length $L$ - optimizing the SnapQueue is a matter of finding the optimal value for $L$. Third, SnapQueue performance depends on the underlying persistent support structure. We described Conc-Trees as a concrete support data structure example with $O(1)$ prepend and append, and $O(\log n)$ concatenation.

Most importantly, we showed that SnapQueue does not incur any performance penalties by having atomic snapshot operations. SnapQueue has similar, and in some cases better, performance compared to other concurrent queues.

This paper revealed several benefits of using persistent data structures when implementing global-state operations. We saw that, in conjunction with an atomic snapshot, it is sufficient to piggy-back the support structure to achieve the desired running time. This indicates that persistent data structures are not relevant only for functional programming, but also crucial for concurrent data structure design.

\section{Acknowledgments}

We would like to thank prof. Doug Lea from SUNY Oswego for his feedback and useful suggestions.

\section{References}

[1] Akka documentation, 2015. http://akka.io/docs/.

[2] SnapQueue Implementation, 2015. https://github.com/stormenroute/reactive-collections/tree/master/reactive-collectionscore.

[3] G. M. Adelson-Velsky and E. M. Landis. An algorithm for the organization of information. Doklady Akademii Nauk SSSR, 146:263-266, 1962.

[4] Y. Afek, N. Shavit, and M. Tzafrir. Interrupting snapshots and the JavaTM size method. J. Parallel Distrib. Comput., 72(7):880-888, July 2012.

[5] E. Allen, D. Chase, J. Hallett, V. Luchangco, J.-W. Maessen, S. Ryu, G. Steele, and S. Tobin-Hochstadt. The Fortress Language Specification. Technical report, Sun Microsystems, Inc., 2007.

[6] N. G. Bronson, J. Casper, H. Chafi, and K. Olukotun. A practical concurrent binary search tree. SIGPLAN Not., 45(5):257268, Jan. 2010.

[7] C. Click. Towards a scalable non-blocking coding style, 2007.

[8] A. Georges, D. Buytaert, and L. Eeckhout. Statistically rigorous java performance evaluation. SIGPLAN Not., 42(10):5776, Oct. 2007.

[9] P. Haller, A. Prokopec, H. Miller, V. Klang, R. Kuhn, and V. Jovanovic. Scala Improvement Proposal: Futures and Promises (SIP-14). 2012.
[10] M. Herlihy and N. Shavit. The Art of Multiprocessor Programming. Morgan Kaufmann Publishers Inc., San Francisco, CA, USA, 2008.

[11] R. Hinze and R. Paterson. Finger trees: A simple generalpurpose data structure. J. Funct. Program., 16(2):197-217, Mar. 2006.

[12] H. Kaplan and R. E. Tarjan. Purely functional representations of catenable sorted lists. In Proceedings of the TwentyEighth Annual ACM Symposium on the Theory of Computing, Philadelphia, Pennsylvania, USA, May 22-24, 1996, pages 202-211, 1996.

[13] D. Lea. Doug Lea's workstation, 2014.

[14] M. M. Michael and M. L. Scott. Simple, fast, and practical non-blocking and blocking concurrent queue algorithms. In PODC, pages 267-275, 1996.

[15] M. Moir and N. Shavit. Concurrent data structures. In Handbook of Data Structures and Applications, D. Metha and S. Sahni Editors, pages 47-14 - 47-30, 2007. Chapman and Hall/CRC Press.

[16] C. Okasaki. Purely Functional Data Structures. Cambridge University Press, New York, NY, USA, 1998.

[17] A. Prokopec. Data Structures and Algorithms for DataParallel Computing in a Managed Runtime. PhD thesis, IC, EPFL, Lausanne, 2014.

[18] A. Prokopec. ScalaMeter Benchmarking Suite Website, 2014. http://scalameter.github.io.

[19] A. Prokopec, N. G. Bronson, P. Bagwell, and M. Odersky. Concurrent tries with efficient non-blocking snapshots. pages 151-160, 2012.

[20] A. Prokopec, H. Miller, T. Schlatter, P. Haller, and M. Odersky. FlowPools: A lock-free deterministic concurrent dataflow abstraction. In $L C P C$, pages 158-173, 2012.

[21] W. Pugh. Concurrent maintenance of skip lists. Technical report, College Park, MD, USA, 1990.

[22] W. N. Scherer, D. Lea, and M. L. Scott. Scalable synchronous queues. Commun. ACM, 52(5):100-111, 2009.

[23] T. Schlatter, A. Prokopec, H. Miller, P. Haller, and M. Odersky. Multi-lane flowpools: A detailed look. Technical report, EPFL, Lausanne, September 2012.

[24] R. Soulé, M. I. Gordon, S. Amarasinghe, R. Grimm, and M. Hirzel. Dynamic expressivity with static optimization for streaming languages. In Proceedings of the 7th ACM International Conference on Distributed Event-based Systems, DEBS '13, pages 159-170, New York, NY, USA, 2013. ACM.

[25] G. Steele. Organizing functional code for parallel execution; or, foldl and foldr considered slightly harmful. International Conference on Functional Programming (ICFP), 2009.

[26] S. Tasharofi. Efficient testing of actor programs with nondeterministic behaviors. $\mathrm{PhD}$ thesis, University of Illinois at Urbana-Champaign, 2014.

[27] M. Thompson, D. Farley, M. Barker, P. Gee, and A. Stewart. Disruptor: High performance alternative to bounded queues for exchanging data between concurrent threads. May 2011. 